\title{
Multivariable decoupling set-point approach applied to a wastewater treat- ment plant
}

\author{
Ramon Vilanova ${ }^{1, a}$ and Orlando Arrieta ${ }^{2}$ \\ ${ }^{1}$ Departament de Telecomunicació i d'Enginyeria de Sistemes, \\ Escola d'Enginyeria, Universitat Autònoma de Barcelona, \\ 08193 Bellaterra, Barcelona, Spain. \\ ${ }^{2}$ Instituto de Investigaciones en Ingeniería, Facultad de Ingeniería, \\ Universidad de Costa Rica, 11501-2060 San José, Costa Rica
}

\begin{abstract}
This paper presents a formulation for the inclusion of the second degree of freedom for MIMO system for decoupling purposes. The proposal is specially effective when combined with decentralized feedback controllers. Loop interaction is of the major problems in the control of MIMO systems, as interaction can be considered as a disturbance coming from all other loops, the design of the decentralized feedback controller is better understood as a disturbance rejection design. In this approach the set-point tracking capabilities may be not as good as expected. The proposed Two-Degree-of-Freedom (2-DoF) formulation provides a complement to the existing controller that can be automatically determined in terms of the available process and feedback controller information.
\end{abstract}

\section{Introduction}

Despite the great developments of advanced process control techniques, [1], [2], it is widely recognized that PI/PID control is still the most commonly adopted control approach in the process industry. The main reason is the fact that this controller is easily understandable and its few parameters have easy interpretation for hand-tuning. This popularity has been inherited in the control of Multi-Input Multi-Output (MIMO) processes, specially for Two-InputTwo-Output (TITO) processes, being decentralized PI/PID controllers the most popular. Within this MIMO context, the decentralized option obviously requires fewer parameters than the full multivariable case. Another side advantage of decentralized PI/PID controllers is that of loop failure tolerance of the resulting closed-loop system [3].

Even the extensive advances on single-loop PI/PID control tuning methods [4-7] all these methods cannot be directly applied to the design of decentralized control systems because of the existence of interaction among loops. Effectively, the presence of interactions among the loops introduce an inherent difficulty to the design of these local controllers. In the presence of strong interactions the effectiveness of the decentralized controllers can be seriously deteriorated or even cause instability. This fact has motivated the extension of single-loop tuning rules to decentralized control systems an active area of research.

A common approach is to tune an individual controller for each loop and then detune each loop by a detuning fac-

\footnotetext{
"Corresponding author: Ramon.Vilanova@uab.cat
}

tor in order to account for interactions. This is the well known Biggest Log modulus (BLT) method of Luyben [8]. Other similar methods [9] design the controllers on the basis of the diagonal elements and do a further detuning on the basis of the RGA elements. Another different approach is to account for loop interactions when designing the individual control loops. In the sequential design method [10], for example, each designed individual loop is closed an subsequent controllers are designed by looking at the generated disturbance. The main drawback of the approach is that the designer has to proceed on a very ad-hoc manner and decisions are taken on the basis of loops already closed. Therefore the order the loops are being designed may have influence on the system performance. Other researches formulate the design problem as an optimization problem by using Linear Matrix Inequalities [11, 12], genetic algorithms [13], Neural Networks [14], Fuzzy approaches [15]. All these methods suffer from the problem of being too much dependent on the objective control function formulated or the order the loops are being closed. These controllers may result in an unstable system under the case of loop-failure or even when the loops are closed in a different order.

A common concern in all these approaches is, in addition to the inherent difficulties of MIMO control, to achieve a suitable trade-off between the disturbance rejection (also needed to minimize process interaction) and the tracking performance. This trade-off is better tackled within a 2-DoF framework. Despite several advanced procedures do exists in literature; see for example the works on [16-18] for 2-DoF controller design on a general set- 
ting, however it is sometimes desirable to keep the design of both degrees of freedom separated and with as much independence as possible. It is in this sense that we propose to add a second degree of freedom for designing a 2-DoF controller. From this alternative perspective, some approaches can be found in the literature aiming at the design of a suitable prefiltering or feedforward control action aimed to improve the set-point following performance of an existing feedback controller. These approaches ranges form the introduction of a reference prefiltering action $[19,20]$ to the design of complementary feedforward control actions: [21].

Even the idea of improving tracking performance by adding complementary parts to an existing controller structure is appealing (it preserves the performance and design principles of the original design) results and can only be found for the Single-Input Single-Output (SISO) case. The only exception is the recent result provided in [22] where an extension of the SISO results of [21] are worked out. The method however requires the solution of a multiobjective optimization problem in order to determine a feasible feedforward control actions. In this paper, a 2-DoF MIMO controller is proposed where the feedback part is assumed to be implemented as a decentralized feedback controller and the part that operates on the prefilter/feedforward paths is conceived with a special structure that allows a possible automatic tuning from the existing feedback control. As it will be seen the resulting overall MIMO controller consists of the same number of elements as that of MIMO controller but distributed along the feedback, prefilter and feedforward terms. Assuming the feedback part is already in place, the determination of the complementary prefilter/feedforward terms will be done by borrowing some recent results on Internal Model Control (IMC) based feedforward control ([23].

The rest of the paper is organized as follows. First of all the problem formulation set up is presented in section 2 , whereas section 3 presents the determination of the decoupling strategy for set-point following on the basis of feedforward control principles. The derivation is first presented for the TITO case and further generalized to a square MIMO system. Section 4 presents some examples of application and section 5 exemplifies the method on a non-linear Activated Sludge Process (ASP). Section 6 closes the paper by presenting main conclusions and outlines possible further developments.

\section{Feedforward based Set-point decoupling 2-DoF controller}

In this section, the feedforward control design ideas presented in [23] will be applied within the context of a multivariable system. In fact, one can see the control signal generated in one of the loops as a disturbance generator for the rest of the loops. Therefore, one possible way of tackling this interaction is by the inclusion of a feedforward control action from one of the loops to the rest in order to attenuate the effect of the existing interaction. In that sense, the application of the ideas proposed in [23] re- sult to a compensation scheme as it is shown in figure (1) for a TITO system. In this figure $Q_{12}^{f f}(s)$ and $T_{12}^{f f}(s)$ are suitable transfer functions (to be defined below) that constitute the feedforward compensation. The same scheme will apply for the compensation that goes from the second to the first loop. In this later case the feedforward blocks will read $Q_{21}^{f f}(s)$ and $T_{21}^{f f}(s)$, but are not shown for clarity.

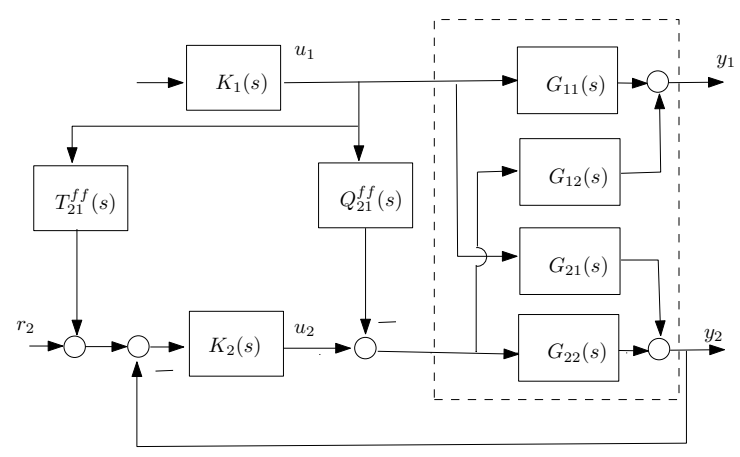

Figure 1. Incorporation of Feedforward corrective actions on a decentralized TITO control scheme.

It is important to note that the application of feedforward control on a single-loop setting has no implications on the stability of the resulting control system (as long as the added blocks are themselves stable). However, within a multivariable approach, like the one concerned here, the addition of these two blocks will introduce new feedback loops that may have repercussions on the final stability. It is therefore needed to workout concrete expressions for these new loops and derive conditions for maintaining stability. The final design of the feedforward terms will therefore need to deal with the unavoidable constraint of maintaining stability and, at the same time, try to improve the attenuation of the interaction effects. Obviously the added stability constraint will make the whole design more complex. In order to avoid this extra complexity and try to have a feedforward approach that is as direct as possible, the following observation is made.

Assume the feedback controllers, $K_{1}(s)$ and $K_{2}(s)$, have been designed on the basis of $G_{1}(s)$ and $G_{2}(s)$ (they can be the direct through transfer functions $G_{11}(s), G_{22}(s)$ or the effective transfer functions if other previously closed loops are taken into account). An estimation of the generated control action on the face of a reference change can be generated by using their associated Internal Model Control (IMC) parameters $Q_{1}(s)$ and $Q_{2}(s)$. It is well known within the IMC framework that the feedback controller and IMC parameter are elated by means of:

$$
\begin{aligned}
K_{i}(s) & =\frac{Q_{i}(s)}{1-G_{i}(s) Q_{i}(s)} \\
Q_{i}(s) & =\frac{K_{i}(s)}{1+G_{i}(s) K_{i}(s)}
\end{aligned}
$$

being the reference to control relation given by $u_{i}=$ $Q_{i}(s) r_{i}$. Therefore, in order to recover a full feedforward action, instead of applying the feedforward compensation 
directly from the control signal, it is proposed to be generated from the corresponding reference signal. On that basis figure (1) is redrawn as figure (2).

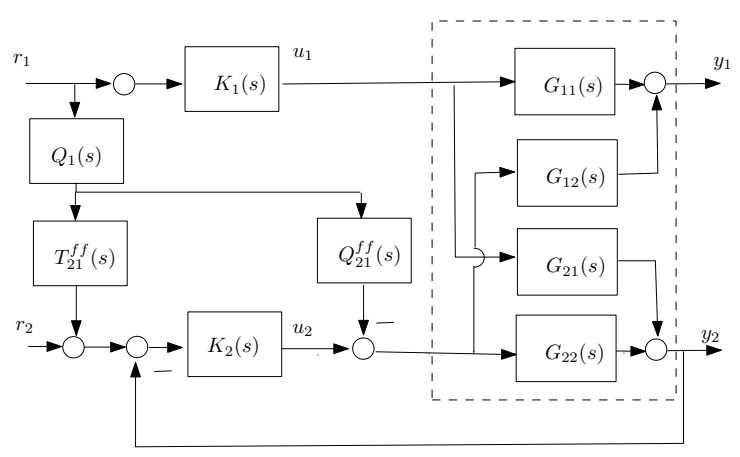

Figure 2. Reference signal based feedforward corrective actions on a decentralized TITO control scheme.

The importance of this change of scenario comes from the generation of the compensating feedforward signal completely from the outside. In this case from the reference signal.

\subsection{Design of the Feedforward Decoupling terms}

Previous section has presented the different terms involved on the feedforward correction that is generated from the first loop into the second loop. Although the same idea applies on the other direction (second loop to the first one), the design equations that follow will only concentrate, for simplicity, on the situation depicted in the figures. Afterwards, a generalization will be presented that will also show how the approach do generalize to a square system of arbitrary dimension.

According to [23], the $Q_{21}^{f f}(s)$ transfer function is, in fact, the feedforward controller to be designed. The design is carried out in two steps

1. Design a feedforward controller, $\overline{Q_{21}^{f f}}(s)$ on the basis of the models, $G_{22}(s)$ and $G_{21}(s)$. This design can be done by trying to approximate the ideal feedforward controller $\overline{Q_{21}^{f f}}(s)=G_{21}(s) / G_{22}(s)$ by existing model matching procedures such as the $\mathcal{H}_{2}$ optimal design of [2] or a min-max approach along the lines of [6]. Here we will use the approach based on [6], where the $\overline{Q_{21}^{f f}}(s)$ is defined as:

$$
\overline{Q_{21}^{f f}}(s)=\arg \min _{Q(s)}\left\|W(s)\left(G_{21}(s)-Q(s) G_{22}(s)\right)\right\|_{\infty}
$$

Ideal feedforward controllers are usually defined in terms of the inverse of the plant. However this usually introduces excessive control actions and high frequency behavior. In turn, this approximate inversion is proposed here where the weighting function $W(s)$ defines the frequency range where the desired inversion error carried out by the feedforward controller is to be penalized.
2. Augment the obtained feedforward controller by a low pass filter $F_{21}^{f f}(s)=1 /\left(\lambda_{21}^{f f} s+1\right)^{n}$ in order to obtain the final feedforward controller as $Q_{21}^{f f}(s)=$ $\overline{Q_{21}^{f f}}(s) F_{21}^{f f}(s)$. The filter order is chosen in order to make the controller transfer function strictly proper. On the other hand, the filter time constant $\lambda_{21}^{f f}$ is chosen in order to tradeoff the reduction of the feedforward control action bandwidth against the loos of achieved nominal performance.

On the other hand, the $T_{21}^{f f}(s)$ term is automatically determined once the feedforward controller $Q_{21}^{f f}(s)$ is calculated. The definition of $T_{21}^{f f}(s)$ is simply as the error incurred by $Q_{21}^{f f}(s)$ on trying to approximate the ideal controller:

$$
T_{21}^{f f}=\left(G_{21}(s)-Q_{21}^{f f}(s) G_{22}(s)\right)
$$

Therefore, problem (3) can alternatively be written as

$$
\left.\overline{Q_{21}^{f f}}(s)=\arg \min _{Q(s)} \| W(s) T_{21}^{f f}(s)\right) \|_{\infty}
$$

\subsection{Generic Feedforward-Decoupling configuration}

The ideas presented in the previous section can be given a more compact form by introducing the following matrices:

$$
\begin{gathered}
K(s)=\left(\begin{array}{cc}
K_{11}(s) & 0 \\
0 & K_{22}(s)
\end{array}\right) \quad Q(s)=\left(\begin{array}{cc}
Q_{11}(s) & 0 \\
0 & Q_{22}(s)
\end{array}\right) \\
Q^{f f}(s)=\left(\begin{array}{cc}
0 & Q_{12}^{f f}(s) \\
Q_{21}^{f f}(s) & 0
\end{array}\right) \quad T^{f f}(s)=\left(\begin{array}{cc}
0 & T_{12}^{f f}(s) \\
T_{21}^{f f}(s) & 0
\end{array}\right)
\end{gathered}
$$

If we now denote the vector signals as $\mathbf{r}=\left(r_{1} r_{2}\right)^{T}$, $\mathbf{u}=\left(\begin{array}{ll}u_{1} & u_{2}\end{array}\right)^{T}$ and $\mathbf{y}=\left(\begin{array}{ll}y_{1} & y_{2}\end{array}\right)^{T}$, we can write:

$$
\begin{aligned}
\mathbf{u} & =K(s)\left(\mathbf{r}+T^{f f}(s) Q(s) \mathbf{r}-\mathbf{y}\right)+Q^{f f}(s) Q(s) \mathbf{r} \\
& =K(s)\left(\left(I+T^{f f}(s) Q(s)\right) \mathbf{r}-\mathbf{y}\right)+Q^{f f}(s) Q(s) \mathbf{r}
\end{aligned}
$$

with

$$
\begin{aligned}
& K(s)=\operatorname{diag}\left\{K_{11}(s), K_{22}(s)\right\} \\
& Q(s)=\operatorname{diag}\left\{Q_{11}(s), Q_{22}(s)\right\}
\end{aligned}
$$

and the $Q^{f f}(s)$ and $T^{f f}(s)$ matrices will be completely offdiagonal matrices defined as:

$$
\begin{aligned}
Q^{f f}(s) & =\left(\begin{array}{cc}
0 & Q_{12}^{f f}(s) \\
Q_{21}^{f f}(s) & 0
\end{array}\right) \\
T^{f f}(s) & =\left(\begin{array}{cc}
0 & T_{12}^{f f}(s) \\
T_{21}^{f f}(s) & 0
\end{array}\right)
\end{aligned}
$$

being each one of the feedforward controllers $Q_{i j}^{f f}(s)$ designed on the basis of: 


$$
\overline{Q_{i j}^{f f}}(s)=\arg \min _{Q(s)}\left\|W_{j}(s)\left(G_{i j}(s)-Q(s) G_{i i}(s)\right)\right\|_{\infty}
$$

therefore

$$
T_{i j}^{f f}(s)=\left(G_{i j}(s)-Q_{i j}^{f f}(s) G_{i i}(s)\right)
$$

Some remarks have to be made with respect to the resulting final controller structure. The design starts from the diagonal matrix $K(s)$, designing an independent controller, $K_{i i}(s)$, for each one of the diagonal process terms $G_{i i}(s)$. Once we have this controller, its corresponding IMC parameter, $Q_{i}(s)$, is computed:

$$
Q_{i i}(s)=\frac{K_{i i}(s)}{1+K_{i i}(s) G_{i i}(s)}
$$

and their associated diagonal matrix, $Q(s)$, can also be generated. On the other hand, the $Q^{f f}(s)$ matrix is fully off-diagonal, having each one of its elements got as the solution to (14). Again, once the $Q_{i j}^{f f}(s)$ are calculated, the $T^{f f}(s)$ matrix can be automatically generated from (15).

\section{Application to an Activated Sludge Process}

The Activated Sludge Process (ASP) is arguably the most popular bioprocess utilized in the treatment of polluted water, using microorganisms present within the treatment plant in the biological oxidation of the wastewater. With the provision of adequate oxygen supply, this process can be maintained to degrade the organic matter in the pollutant. Most modern wastewater treatment plants is of this type and consists of a series of bioreactors and settlers. In this report the configuration of a single bioreactor connected to a single secondary clarifier is considered. See fig. (3). The simplified but still realistic and highly non-linear four-state multivariable model considered here is the Activated Sludge Process (ASP) [24].

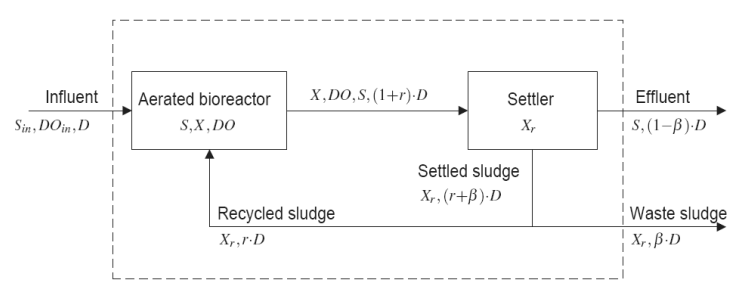

Figure 3. Activated Sludge Process layout

\subsection{Activated Sludge Process (ASP) Description}

The mathematical model considered in this paper is given in [24]. The ASP process comprises an aerator tank where microorganisms act on organic matter by biodegradation, and a settler where the solids are separated from the wastewater and recycled to the aerator. The layout is shown in figure (3). The component balance for the substrate, biomass, recycled biomass and dissolved oxygen provide the following set of non-linear differential equations:

$$
\begin{aligned}
\frac{d X(t)}{d t} & =\mu(t) X(t)-D(t)(1+r) X(t)-r D(t) X_{r}(t)(17) \\
\frac{d S(t)}{d t} & =-\frac{\mu(t)}{Y} X(t)-D(t)(1+r) S(t)+D(t) S_{i n}(18) \\
\frac{d D O(t)}{d t} & =-\frac{K_{o} \mu(t)}{Y} X(t)-D(t)(1+r) D O(t) \\
& +K_{L a}\left(D O_{s}-D O(t)\right)+D O(t) D O_{i n} \\
\frac{d X_{r}(t)}{d t} & =D(t)(1+r) X(t)-D(t)(\beta+r) X_{r}(t)
\end{aligned}
$$

$$
\mu(t)=\mu_{\max } \frac{S(t)}{k_{S}+S(t)} \frac{D O(t)}{k_{D O}+D O(t)}
$$

where $X(t)$ - biomass, $S(t)$ - substrate, $D O(t)$ - dissolved oxygen, $D O_{s}$ - maximum dissolved oxygen, $X_{r}(t)$ - recycled biomass, $D(t)$ - dilution rate, $S_{i} n$ and $D O_{i} n$ - substrate and dissolved oxygen concentrations in the influent, $Y$ - biomass yield factor, $\mu$ biomass growth rate, $\mu_{\max }$ - maximum specific growth rate, $k_{S}$ and $k_{D O}$ - saturation constants, $K_{L a}=\alpha W$ - oxygen mass transfer coefficient, $\alpha$ - oxygen transfer rate, $W$ - aeration rate, $K_{o}$ model constant, $r$ and $\beta$ - ratio of recycled and waste flow to the influent. The model parameterization is according to tables (1) and (2). On the other hand,the influent concentrations are set to $S_{\text {in }}=200 \mathrm{mg} / \mathrm{l}$ and $D O_{\text {in }}=0.5 \mathrm{mg} / \mathrm{l}$.

\begin{tabular}{ll}
\hline Biomass & $X(0)=215 \mathrm{mg} / \mathrm{l}$ \\
Substrate & $S(0)=55 \mathrm{mg} / \mathrm{l}$ \\
Dissolved Oxygen & $D O(0)=6 \mathrm{mg} / \mathrm{l}$ \\
Recycled Biomass & $X_{r}(0)=400 \mathrm{mg} / 1$ \\
\hline
\end{tabular}

Table 1. Initial Contitions

\begin{tabular}{ll}
\hline$\beta=0.2$ & $K_{c}=2 \mathrm{mg} / \mathrm{l}$ \\
$r=0.6$ & $K_{s}=100 \mathrm{mg} / \mathrm{l}$ \\
$\alpha=0.018$ & $K_{D O}=0.5$ \\
$Y=0.65$ & $D O_{s}=0.5 \mathrm{mg} / 1$ \\
$\mu_{\text {max }}=0.15 h^{-1}$ & \\
\hline
\end{tabular}

Table 2. Kinetic parameters

With respect to the control problem definition, the waste water treatment process is considered under the assumption that the dissolved oxygen, $D O(t)$, and substrate, $X(t)$, are the controlled outputs of the plant, whereas the dilution rate, $D(t)$, and aeration rate $W(t)$ are the two manipulated variables.

\subsection{Linearized model}

For controller design purposes, the previous model is linearized around the operating point defined by the steady-state inputs of $D_{s s}=0.0825$ and $W_{s s}=90$. The resulting linear model will have a transfer function matrix of the form:

such that

$$
\left(\begin{array}{c}
S(t) \\
D O(t)
\end{array}\right)=\left(\begin{array}{cc}
G_{11}(s) & G_{12}(s) \\
G_{21}(s) & G_{22}(s)
\end{array}\right)\left(\begin{array}{c}
D(t) \\
W(t)
\end{array}\right)
$$

The $G_{i j}(s)=n_{i j}(s) / d(s)$ transfer function components are given as: 


$$
\begin{aligned}
G_{11}(s) & =\frac{134.0243 s^{3}+295.3529 s^{2}+53.5176 s+.5855}{s^{4}+2.4617 s^{3}+0.9859 s^{2}+0.1107 s+0.0008} \\
G_{12}(s) & =\frac{-0.0312 s^{2}-0.0062 s-0.0001}{s^{4}+2.4617 s^{3}+0.9859 s^{2}+0.1107 s+0.0008} \\
G_{21}(s) & =\frac{-9.2834 s^{3}-15.0312 s^{2}-2.6325 s-0.0123}{s^{4}+2.4617 s^{3}+0.9859 s^{2}+0.1107 s+0.0008} \\
G_{22}(s) & =\frac{0.0699 s^{3}+0.0340 s^{2}+0.0042 s+2.910^{-5}}{s^{4}+2.4617 s^{3}+0.9859 s^{2}+0.1107 s+0.0008}
\end{aligned}
$$

\subsection{Decentralized PI control}

As a first step, two PI feedback controllers are designed. The design is based on the 2DoF PI tuning approach presented in [25] and within the ASP process in [26]. Due to space constraints, just the tuning for both controllers is provided as well as the time responses achieved for such tuning in comparison with a well known multivariable PID technique such as that of [27]. The resulting PI tuning parameters are: $K_{p 1}=0.006, T_{i 1}=3.0$ and $\beta_{1}=0.67$ for substrate loop, whereas $K_{p 2}=3.13, T_{i 2}=0.8$ and $\beta_{2}=1$ ar got for the dissolved oxygen loop.
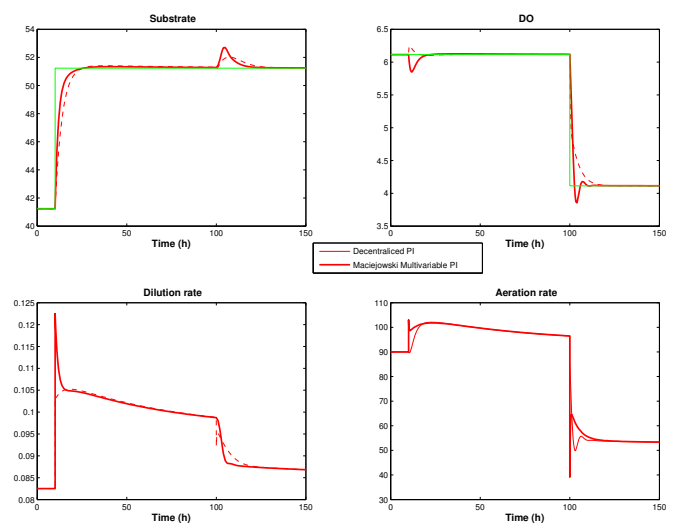

Figure 4. Comparison of the decentralized PI tuning and the Multivariable PI method of Maciejowski

Now, in order to improve the performance where set-point changes are applied, the proposed feedforward decoupling controllers are applied. In this case, as $G_{22}(s)$ and $G_{21}(s)$ share the same denominator a straightforward choice for $Q_{21}^{f f}$ results as:

$$
Q_{21}^{f f}=\frac{n_{22}(s)}{n_{21}(s)} \frac{1}{\left(\lambda_{21}^{f f} s+1\right)}
$$

where $\lambda_{21}^{f f}$ is the tuning parameter associated with the feedforward compensator. The tuning of this parameter can be done by observing it has to be in accordance with the expected control signal bandwidth. This way, the poles of $Q_{11}(s)$ (the IMC parameter of $\left.K_{11}(s)\right)$ are computed and $\lambda_{21}^{f f}$ is chosen, for example, five times smaller than the corresponding fastest time constant of $Q_{11}(s)$. By applying such simple rule the following values are obtained for the feedforward filter time constants: $\lambda_{21}^{f f}=0.4$ and $\lambda_{12}^{f f}=0.2$. The resulting improvement in interaction compensation is shown in figure (5). The figure axis have been magnified in order to have a better look at the difference with respect to the purely decentralized control case. It is important to notice that the change incurred in the corresponding control actions it is not really large and basically introduced anticipatory control action (as expected).

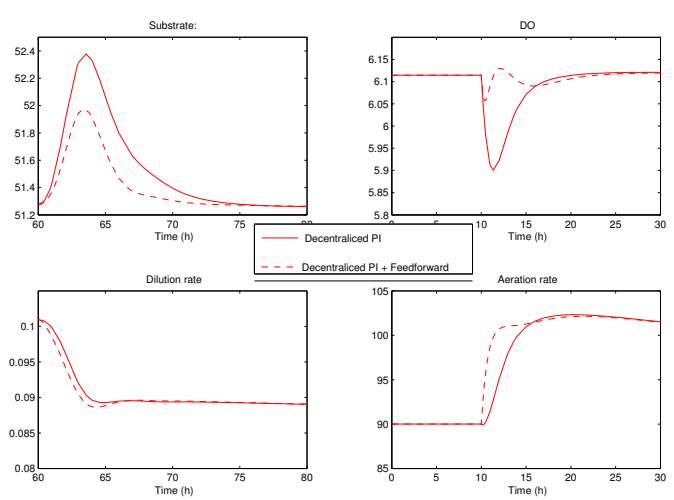

Figure 5. Interaction reduction by using the reference-driven feedforward actions.

It is important to remark that the generated feedforward signals are based on:

- The linear models of the process, therefore only retaining local information.

- The generation of the expected control signal from the applied reference input. This generation is also performed on the basis of linear models.

However, as it is shown in figure (6) if we compare the performance of the feedforward corrections by directly using the control signal or by using the proposed generation from the reference signal, it is seen that both performances are comparable and, in some cases, even better for the reference-driven case.
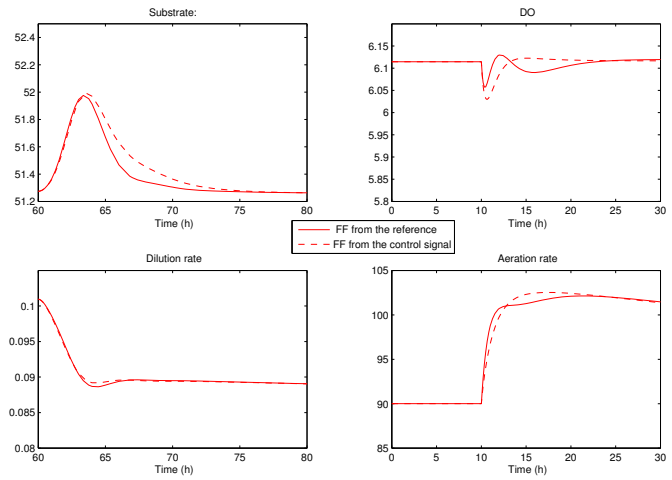

Figure 6. Comparison of the reference-driven and control signaldriven feedforward actions.

\section{Conclusions}

This paper has presented a formulation for the incorporation of feedforward control action from the reference signal in multivariable control in order to alleviate the effects of process interaction and improve the performance for set-point following. 
The approach has special appealing for decentralized PI/PID control based on IMC-like tuning methods. In such cases, the tuning is directed by the desired closed-loop bandwidth. It is this parameter that is used for the tuning of the feedforward filters. The overall resulting control configuration has the same components as a full multivariable controller. However just the diagonal part of the controller remains within the loop, whereas the rest is located outside. Therefore there is no need to incorporate additional stability considerations.

Future efforts are directed towards a simultaneous design of the feedback and feedforward parts, as well as the exploration of possibilities regarding the inclusion of such feedforward actions within the loop and its use for interaction effects attenuation also when dealing with external disturbances.

\section{Acknowledgments}

The financial support from the University of Costa Rica, under the grants 322-B4-218 and 731-B4-213, is greatly appreciated. Also, this work has received financial support from the Ministerio de Economia e Innovacion of Spain under project DPI201347825-C3-1-R.

\section{References}

[1] E. Camacho, C. Bordons, Model Predictive Control in the Process Industry (Springer-Verlag, 1995)

[2] M. Morari, E. Zafirou, Robust Process Control. (Prentice-Hall International, 1989)

[3] S. Skogestad, M. Morari, Automatica 25, 119 (1989)

[4] S. Skogestad, Modeling, Identification and Control 25(2), 85 (2004)

[5] K. Astrom, T. Hagglund, Journal of Process Control 14, 635 (2004)

[6] R. Vilanova, Journal of Process Control 18, 61 (2008)

[7] K. Åström, T. Hägglund, Advanced PID Control (ISA - The Instrumentation, Systems, and Automation Society, 2006)

[8] W. Luyben, Ind Eng. Chem Des. Dev. 25, 654 (1986)

[9] I. Chien, H. Huang, J. Yang, Ind. Eng. Chem. res. 37 (1999)
[10] M. Hovd, S. Skogestad, Automatica 30, 1601 (1994)

[11] J. Bao, J. Forbes, P.J., Ind. Eng. Chem. res. 38 (1999)

[12] S. Tong, Q. Zhang, Int. J. Innovative Computing, Information and Control 4, 3385 (2008)

[13] C. Vlachos, D. Williams, J. Gomm, IEE- Proc on Control Theory and Applications 146 (1999)

[14] Y. Abe, J.I. M. Konishi, R. Hasagawa, M. Watanabe, H. Kamijo, Int. J. Innovative Computing, Information and Control 4, 2649 (2008)

[15] S. Tong, W. Wang, L. Qu, Int. J. Innovative Computing, Information and Control 3, 657 (2007)

[16] M.J. Grimble, Robust Industrial Control. Optimal design Approach for Polynomial Systems (PrenticeHall International, 1994)

[17] D. Limebeer, E. Kasenalli, J. Perkins, Automatica 29, 157 (1993)

[18] R. Vilanova, I. Serra, C. Pedret, R. Moreno, IETControl Theory and Applications 1, 1322 (2007)

[19] A. Leva, L. Bascetta, Automatica 11, 1984 (2007)

[20] L. Bascetta, A. Leva, Journal of Process Control 18, 465 (2008)

[21] A. Visioli, Journal of Process Control 14, 457 (2004)

[22] S. Piccagli, A. Visioli, Journal of Process Control 19, 978 (2009)

[23] R. Vilanova, O. Arrieta, P. Ponsa, ISA- Transactions 48, 439 (2009)

[24] F. Nejjari, A. Benhammou, B. Dahhou, G. Roux, Int. J. Adapt. Control Signal Process. pp. 347-365 (1999)

[25] V. Alfaro, R. Vilanova, O. Arrieta, Analytical Robust Tuning of PI Controllers for First-Order-PlusDead-Time Processes, in 13th IEEE International Conference on Emerging Technologies and Factory Automation (2008), Hamburg-Germany

[26] R. Vilanova, R. Katebi, V. Alfaro, Multi-loop PIbased control strategies for the Activated Sludge Process, in 14th IEEE International Conference on Emerging Technologies and Factory Automation (2009), Mallorca, Spain

[27] J.M. Maciejowski, 1st edition, Addison Weslew, Wokingham (1989) 